occurred. These hiatuses impart a statistical bias that causes sediment accumulation rates to seem greater when calculated from measurements taken at shorter time intervals ${ }^{10}$. Indeed, geochemical studies suggest that the delivery of continent-derived sediment to the global ocean has essentially remained constant over the past approximately 10 million years ${ }^{11}$.

This statistical pitfall can be overcome by directly assessing the thermal history of rocks. Rocks in the crust cool as overlying material is removed by erosion at the surface (a process referred to as exhumation), because they are brought nearer to the surface. Several minerals in bedrock contain trace amounts of radioactive isotopes, whose decay generates daughter products. The ability of minerals to retain these products in their crystal lattices depends on temperature, and so the amount of a daughter product that has accumulated in a mineral can be used to work out the time passed since the mineral was at a specific temperature (the blocking temperature). This forms the basis of a technique known as thermochronology. By analysing mineral systems that have different retentivities, the rate at which rock cooled can be estimated.

Herman and colleagues took this idea a step further by developing a method that works out the temperature and exhumation histories that best fit thermochronologic data obtained for multiple rock samples collected within close proximity. This approach assumes that nearby samples have similar exhumation histories, and thus that any differences in thermochronologic age between mineral systems with different blocking temperatures are explained by the systems having different cooling rates over time. For the past five years, this work has stood as the most compelling evidence that the cooling of global climates drove synchronous increases in erosion rate as a direct consequence of the growth of mountain glaciers.

Schildgen et al. now demonstrate that the assumption that nearby samples have similar exhumation histories is often not appropriate. In nearly all of the regions that were thought to exhibit an increased exhumation rate over time ${ }^{2}$, samples that have been recently exhumed are juxtaposed with older samples at geological boundaries. In the Western Alps, for instance, an abrupt difference in thermochronometer age for samples collected across a fault system reflects the different erosional histories for rocks on either side of the fault. Yet when these data are combined using Herman and colleagues' data-analysis method, the age difference is transformed into an apparent increase in average erosion rate through time for the entire region. Perhaps even more convincingly, Schildgen and colleagues used artificial data sets constructed to represent known geological scenarios to demonstrate that spurious results can occur when data are combined from samples subjected to erosion that was constant in time, but non-uniform in space.

The authors evaluated the other 29 examples of apparent accelerated erosion presented by Herman et al., and found that nearly threequarters could have resulted from the combination of data from samples that have different erosion histories. They argue that a few of the remaining cases reflect changes in tectonic boundary conditions (changes in fault activity). Only three of the original study sites seem to have experienced climatically driven acceleration in cooling, and each of these was associated with the formation of deep valleys by glaciers (Fig. 1). This re-evaluation suggests that, although glaciers can be an effective agent of landscape erosion, the thermochronology methods used by Herman et al. don't have the resolution required to assess whether global increases in glacial erosion occurred during the past few million years.

The finding that some sites do seem to reflect enhanced erosion by glacial ice, however, suggests that the story is not yet complete. As the precision and sensitivity to lower temperatures (shallower depths in Earth's crust) of thermochronology continue to improve, the method might be able to constrain estimates of changes in erosion over relatively short timescales ${ }^{12}$. Similar improvements in the precision with which the age of coarse sediments derived from glacial erosion can be determined ${ }^{13}$ might also help to resolve questions of how rapidly such deposits accumulated.

Schildgen and colleagues' analysis reminds us that deformation of Earth's rigid outer shell matters: it deforms in a brittle way, breaking along geological faults that, in turn, produce boundaries between regions that have different rates of uplift. The resulting mountain ranges are subject to localized differences in exhumation. The authors' findings also teach us a broader lesson: as we use increasingly sophisticated analyses of 'big data' to gain insight into global trends in geology, we must not lose sight of the physical processes that operate locally. Incorporating site-specific geological constraints into analyses of global data sets will be essential in future studies.

Knowledge of how Earth responded to past climate change continues to be relevant to our future. Schildgen and colleagues' work removes one of the remaining pillars that supported the hypothesis that cooling climates were responsible for a global acceleration in erosion. The idea that erosion rates over the past few million years might have been less sensitive to cooling than was thought challenges us to re-examine our long-held notions of how erosion and climate systems interact. .

Eric Kirby is in the College of Earth, Ocean and Atmospheric Sciences, Oregon State University, Corvallis, Oregon 97330, USA. e-mail:eric.kirby@oregonstate.edu

1. Raymo, M. E. \& Ruddiman, W. F. Nature 359, 117-122 (1992).

2. Herman, F. et al. Nature 504, 423-426 (2013).

3. Schildgen, T. F., van der Beek, P. A., Sinclair, H. D. \& Thiede, R. C. Nature 559, 89-93 (2018).

4. Berner, R. A., Lasaga, A. C. \& Garrels, R. M. Am. J. Sci. 283, 641-683 (1983).

5. Raymo, M. E., Ruddiman, W. F. \& Froelich, P. N. Geology 16, 649-653 (1988).

6. Zachos, J., Pagani, M., Sloan, L., Thomas, E. \& Billups, K. Science 292, 686-693 (2001)

7. Hay, W. W., Sloan, J. L. II \& Wold, C. N. J. Geophys. Res. 93, 14933-14940 (1988).

8. Peizhen, Z., Molnar, P. \& Downs, W. R. Nature 410 891-897 (2001).

9. Willett, S. D. Annu. Rev. Earth Planet. Sci. 38 411-437 (2010).

10.Schumer, R. \& Jerolmack, D. J. J. Geophys. Res. Earth Surf. 114, F00A06 (2009).

11. Willenbring, J. K. \& von Blanckenburg, F. Nature 465, 211-214 (2010)

12. King, G. E., Herman, F. \& Guralnik, B. Science 353, 800-804 (2016).

13.Balco, G. \& Shuster, D. L. Earth Planet. Sci. Lett. 286 570-575 (2009).

\title{
Actin proteins assemble to protect the genome
}

\begin{abstract}
The assembly of polymerized actin with motor proteins at DNA breaks in the nucleus supports the mobility and repair of DNA. This finding reveals a layer of regulation that helps to preserve genome integrity. SEE ARTICLES P.54 \& P.61
\end{abstract}

\section{VASSILIS ROUKOS}

$\mathrm{T}$ The protein actin polymerizes to produce filaments that form crosslinked networks in the cytoplasm of cells. These networks support many fundamental biological processes - such as cell movement and division and the intracellular trafficking of molecules. Reports that actin also has functions in the cell nucleus remain controversial ${ }^{1,2}$, partly because of the challenges of performing experiments that exclusively perturb the nuclear actin pool without also perturbing actin in the cytoplasm. Two studies ${ }^{3,4}$ in this issue now provide the most compelling evidence so far that polymerized actin has roles in cell nuclei in which DNA has been damaged, and that it could be essential for maintaining genome stability.

In cell nuclei, DNA is packaged with 


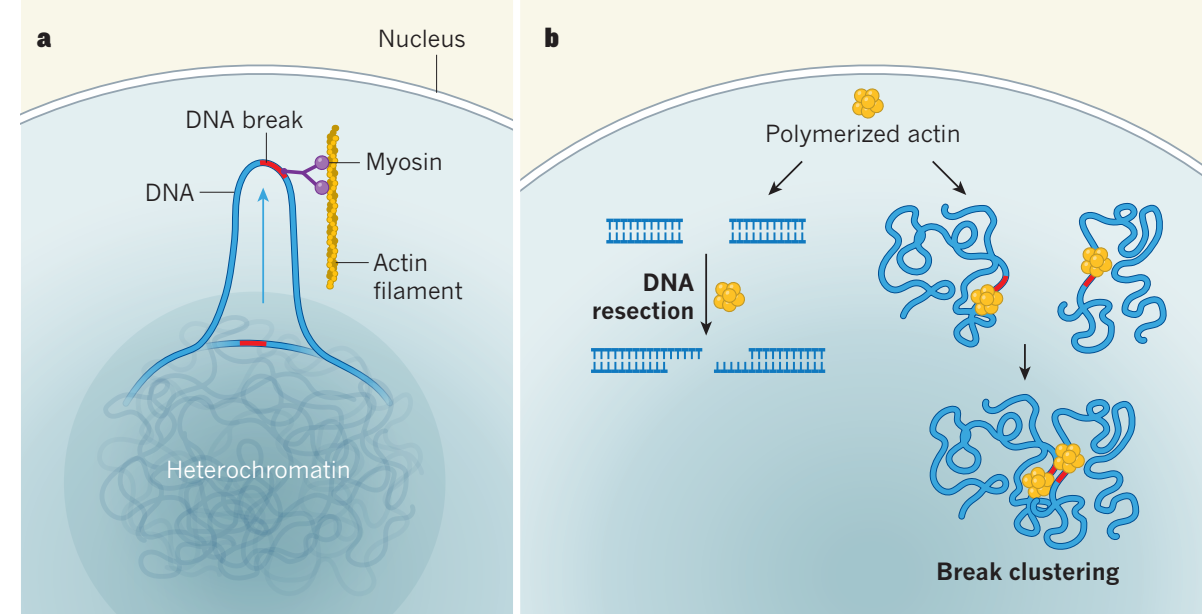

Figure 1 | Nuclear actin polymerizes to preserve genome stability. DNA is packaged with proteins in the nucleus to form a material called chromatin, which is compartmentalized into different domains, including heterochromatin. a, Caridi et al. ${ }^{3}$ report that, in cells of the fruit fly Drosophila melanogaster, DNA breaks in heterochromatin are moved to the nuclear periphery to ensure correct repair - motor proteins called myosins 'walk' the DNA breaks along filaments made from polymerized actin protein. b, Schrank et al. ${ }^{4}$ report that, in human cells, polymerized actin promotes the processing (resection) of broken DNA ends in chromatin (the compartments involved were not determined) and facilitates a DNArepair pathway called homologous recombination (HR, not shown), although the underlying mechanism is unclear. Polymerization of actin also increases the mobility of DNA breaks that will be repaired by HR and the ability of a subset of DNA breaks to form clusters in the nucleus. Prevention of any of the processes shown in $\mathbf{a}$ and $\mathbf{b}$ leads to repair defects and increased genomic instability.

proteins to form a material called chromatin, which is subdivided into different domains. One of these domains, known as heterochromatin, is a tightly packed form of DNA characterized by large segments of repeated DNA sequences. These are highly prone to an aberrant form of genome shuffling called ectopic recombination, and therefore present a serious threat to genomic integrity. In fruit flies and mice, heterochromatic DNA that contains double-strand breaks (DSBs) is moved outside this domain to prevent such aberrant recombination $^{5-7}$, but the mechanism involved has not been known. Actin filaments form in the nuclei of mammalian cells in response to DNA damage $^{8,9}$, but their function in DNA repair has also been unclear.

Caridi et al. ${ }^{3}$ (page 54) show that a subset of DSBs in fruit-fly cells move from heterochromatin towards the nuclear periphery in long-lasting, directed motions, rather than along random paths. Such directed motion has previously been observed only for a handful of cases in the nuclei of mammalian cells ${ }^{10-12}$. Crucially, the authors provide insight into how this relocation occurs. They find that nuclear actin polymerizes to form filaments at heterochromatic DSB-repair sites, in a process that requires the presence of the protein complex Arp 2/3 and its activators (the Scar and Wash proteins). They observe that Arp $2 / 3$ promotes the formation of actin filaments that grow from heterochromatic DSBs towards the nuclear periphery. The nuclear motor proteins myo$\sin \mathrm{I}$ and myosin V then 'walk' the repair sites along the actin filaments (Fig. 1a). This walk is triggered by the myosin-activating protein
Unc45 after filament formation, indicating that relocation is regulated both in space and in time.

An important finding of Caridi and colleagues is that recruitment of Arp2/3 and myosins at heterochromatic DSBs requires the DSB-repair protein Mre11 and a heterochromatin component, the HP1a protein. This suggests that DSB detection or processing is required for the recruitment of the motor proteins to the damaged sites and reveals why only heterochromatic DSBs are relocated to the nuclear periphery. Notably, the authors demonstrate that, when nuclear actin and myosins are defective in both fruit-fly and mouse cells, the integrity of the genome in heterochromatin is impaired and cells become less able to survive DNA damage, suggesting that actin and myosin have key roles in maintaining genome stability in the nucleus.

DSB repair can proceed by two distinct mechanisms: the error-prone non-homologous end-joining pathway (NHEJ) and the usually error-free homologous recombination (HR) pathway. During NHEJ, broken ends of DNA are simply re-stitched together, whereas $\mathrm{HR}$ requires that DNA ends are first processed to generate stretches of single-stranded DNA, which then search for appropriate (homologous) DNA sequences to use as a template for repair. Schrank et al. ${ }^{4}$ (page 61) report that polymerization of nuclear actin in human cells is specifically required for the efficient repair of DSBs by HR.

Using a cellular system that controllably induces DSBs mainly within euchromatin ${ }^{13}$, the authors find that - as in heterochromatic breaks - Arp $2 / 3$ is recruited to the damaged genomic sites. However, the Arp $2 / 3$ activators recruited to these breaks are different from the ones shown by Caridi et al. to be recruited at heterochromatic breaks. This raises the possibility that different regulatory mechanisms promote actin-filament formation in different genomic and chromatin contexts.

Intriguingly, Schrank and colleagues find that Arp2/3 is recruited only at DSBs undergoing HR, whereas the Wiskott-Aldrich syndrome protein (WASP, an Arp2/3 activator) is recruited at breaks repaired by both $\mathrm{HR}$ and NHEJ. This observation indicates the existence of a currently unclear regulation mechanism that promotes actin polymerization so that it occurs specifically at breaks undergoing HR. The researchers also show that inhibition of nuclear-actin polymerization leads to a decrease in the processing of DNA ends and in the efficiency of $\mathrm{HR}$ repair, placing nuclear actin at the core of the cellular response to DNA damage (Fig. 1b).

Schrank and co-workers' efforts might turn out to be relevant to disease. Wiskott-Aldrich syndrome (WAS) is a disorder characterized by severe immunodeficiency and predisposition to cancers caused by mutations in the WAS gene ${ }^{14}$ (which encodes WASP). How WAS mutations cause malignancies is largely unknown at the molecular level. Schrank et al. report that inhibition of WASP leads to defects in HR repair, and that immune cells (lymphocytes) from people with WAS show evidence of defects in DNA-end processing and have higher sensitivity to DNA-damaging agents than do lymphocytes from healthy people. Future studies should investigate whether the repair defects associated with WASP malfunction in the nucleus contribute to the malignancies in people with WAS.

The authors further show that perturbations of actin polymerization in the nucleus lead to a decrease in the mobility of DSBs, alongside the repair defects. This reduced motion contributes to a reduction in the ability of a subset of DSBs to form clusters within the nucleus. The authors propose that this reduced clustering limits HR efficiency by decreasing the local concentration of factors that process DNA ends. Given that relatively few DSBs form clusters, another plausible scenario is that actin-dependent DSB movement and DNAend processing are parallel events, and are not functionally linked.

The two new studies provide a framework for further investigations into the role of nuclear polymerized actin in the cell's response to DNA damage. Although the key biological players that support the nuclear functions of actin have been identified, the physical connections that attach damaged chromatin to nuclear actin structures and to motor proteins are unknown. The next step should be to use super-resolution microscopy and other imaging tools to fully characterize 
actin structures in the nucleus and to discover how the architecture of chromatin and the nucleus affects the formation and remodelling of actin polymerization. Further studies are also required to unravel the mechanism by which polymerized actin facilitates chromatin mobility and DNA-end processing. Given that genomic instability is a major contributor to the development of cancer, working out how cells use actin to safeguard their genome will have implications for our understanding of the basic principles of cancer aetiology.
Vassilis Roukos is at the Institute of

Molecular Biology, 55128 Mainz, Germany.

e-mail:v.roukos@imb-mainz.de

1. de Lanerolle, P. \& Serebryannyy, L. Nature Cell Biol. 13, 1282-1288 (2011).

2. Belin, B. J. \& Mullins, R. D. Nucleus 4, 291-297 (2013).

3. Caridi, C. P. et al. Nature 559, 54-60 (2018).

4. Schrank, B. R. et al. Nature 559, 61-66 (2018),

5. Chiolo, I. et al. Cell 144, 732-744 (2011).

6. Tsouroula, K. et al. Mol. Cell 63, 293-305 (2016).

7. Jakob, B. et al. Nucleic Acids Res. 39, 6489-6499 (2011).
8. Belin, B. J., Lee, T. \& Mullins, R. D. eLife 4, e07735 (2015).

9. Andrin, C. et al. Nucleus 3, 384-395 (2012)

10.Chuang, C. H. et al. Curr. Biol. 16, 825-831 (2006).

11.Dundr, M. et al. J. Cell Biol. 179, 1095-1103 (2007).

12.Cho, N. W., Dilley, R. L., Lampson, M. A. \& Greenberg, R. A. Cell 159, 108-121 (2014).

13.Aymard, F. et al. Nature Struct. Mol. Biol. 4, 366-374 (2014).

14. Massaad, M. J., Ramesh, N. \& Geha, R. S. Ann. NY Acad. Sci. 1285, 26-43 (2013).

This article was published online on 20 June 2018.

\section{Picturing a key brain protein}

\begin{abstract}
Proteins called $\mathrm{GABA}_{\mathrm{A}}$ receptors have a pivotal role in neuronal inhibition, and are targets of several drugs. Structures of the most abundant $\mathrm{GABA}_{\mathrm{A}}$ receptor in humans are now reported, and will aid future drug discovery. SEE ARTICLE P.67
\end{abstract}

\section{ERWIN SIGEL}

$\mathrm{T}$ The daytime biological activity of humans is based on a delicate equilibrium between excitatory and inhibitory signalling in the brain. The neurotransmitter molecule that mediates the main inhibitory signal is $\gamma$-aminobutyric acid (GABA), which binds to and activates ion channels, including a family known as $\mathrm{GABA}_{\mathrm{A}}$ receptors ${ }^{1}$ found in the cell membranes of neurons. The activity of these receptors is modulated by many drugs ${ }^{2}$, including sedatives, anxiolytics and sleeping pills. $\mathrm{GABA}_{\mathrm{A}}$ receptors can be assembled from several types of subunit, and the most abundant $\mathrm{GABA}_{\mathrm{A}}$ receptors in the adult human brain consist of two $\alpha 1$ subunits, two $\beta 2$ subunits and one $\gamma 2$ subunit. On page 67 , Zhu et al. ${ }^{3}$ present two structures of this receptor isoform in complex with GABA and a drug molecule known as flumazenil, providing much-needed insight that will aid future drug-discovery efforts.

The $\mathrm{GABA}_{\mathrm{A}}$ receptors that mediate fast signalling are found in several locations, most importantly at the synaptic junctions between neurons. Once activated by GABA, the channels in the receptors open, and conduct chloride ions through their pores. Benzodiazepines are a widely used class of drug that binding to a specific site ${ }^{4}$ distinct from that at which GABA binds. These compounds do not induce receptor activity on their own, but increase activity triggered by GABA, a process

Zhu et al. prepared the receptors for their study by expressing them in human embryonic kidney (HEK) cells in the presence of GABA acts at some subtypes of $\mathrm{GABA}_{\mathrm{A}}$ receptor by known as positive allosteric modulation. and flumazenil, which is an antagonist for the benzodiazepine binding site (that is, it blocks the site). The authors used cryo-electron microscopy to obtain two high-resolution structures of the synaptic $\alpha 1 \beta 2 \gamma 2 \mathrm{GABA}_{\mathrm{A}}$ receptor (see Fig. 1 of the paper ${ }^{3}$ ), which they propose are in non-conducting, desensitized states - closed states of the channel that occur when the receptors have had prolonged exposure to GABA. Crucially, the structure confirms that the number of each subunit type and the arrangement of the subunits in to what had previously been postulated ${ }^{1}$.

The structures reveal differences between the GABA binding sites (which are found at the two $\beta 2-\alpha 1$ subunit interfaces in the receptor), the benzodiazepine binding site (which is found at the $\alpha 1-\gamma 2$ interface, at an equivalent position to the GABA binding site), and equivalent sites at the $\alpha 1-\beta 2$ and $\gamma 2-\beta 2$ interfaces to which neither GABA nor benzodiazepines bind (Fig. 1). These differences help to explain why the different sites bind (or do not bind) GABA or benzodiazepines. Both the $\gamma 2-\beta 2$ and $\alpha 1-\beta 2$ sites are potential targets for drug discovery. A compound that binds at the $\alpha 1-\beta 2$ interface has already been reported ${ }^{5}$, but no compounds have yet been found for $\gamma 2-\beta 2$. The new receptor structures will provide a the assembled pentameric receptor correspond

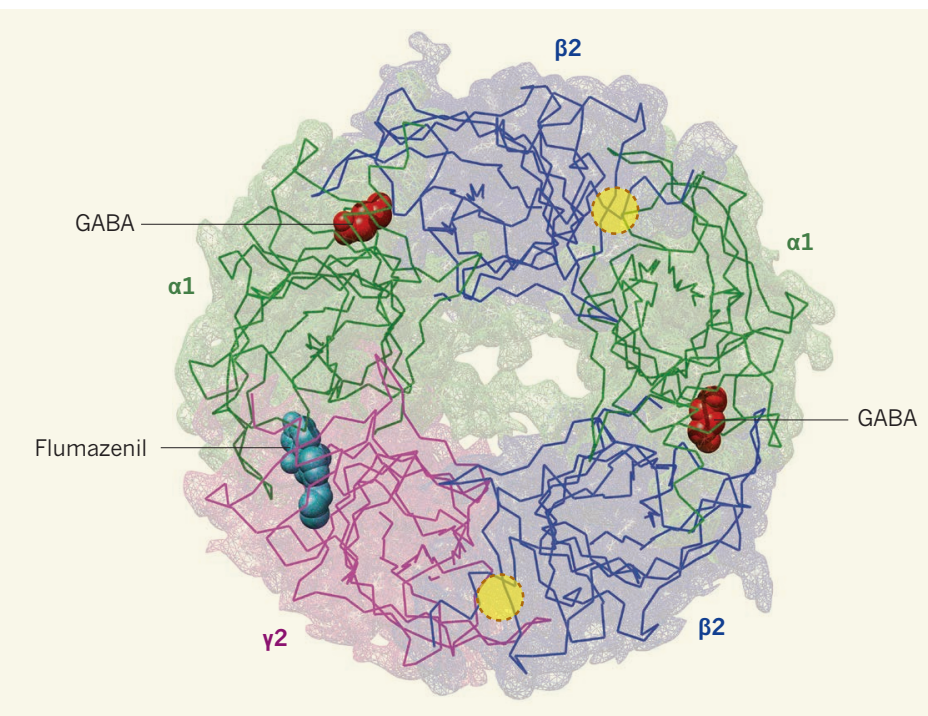

Figure 1 | Structure of the $\alpha 1 \beta 2 \gamma 2 \mathrm{GABA}_{\mathrm{A}}$ receptor viewed from the synapse. $\mathrm{GABA}_{\mathrm{A}}$ receptors are involved in neuronal inhibition and are targeted by several drugs. They form channels in the membranes of neurons, at different locations, most importantly at the synaptic junctions that connect pairs of neurons. Zhu et al. ${ }^{3}$ report the structure of the most abundant $\mathrm{GABA}_{\mathrm{A}}$ receptor found in adult humans, which consists of two $\alpha 1$ subunits, two $\beta 2$ subunits and one $\gamma 2$ subunit. A molecule of $\gamma$-aminobutyric acid (GABA) - the naturally occurring ligand that activates the receptors - is bound at each of the GABA-binding sites, which are located at the two $\beta 2-\alpha 1$ subunit interfaces. The drug flumazenil is bound at the benzodiazepine site at the $\alpha 1-\gamma 2$ subunit interface (the site at which drug molecules from the benzodiazepine class bind). The $\gamma 2-\beta 2$ and $\alpha 1-\beta 2$ interfaces contain pockets (yellow circles) that are at equivalent positions to the GABA and benzodiazepine sites, and which might be suitable targets for drug discovery. (Adapted from Fig. 2c of ref. 3.) 